\title{
DIAGRAMA DE MANEJO DA DENSIDADE PARA Pinus taeda NO ESTADO DO PARANÁ
}

\author{
Fabiane Aparecida de Souza Retslaff ${ }^{1 *}$, Afonso Figueiredo Filho ${ }^{1}$, Sebastião do Amaral Machado ${ }^{2}$, \\ Julio Eduardo Arce ${ }^{2}$, Eldemar Jaskiu ${ }^{3}$ \\ ${ }^{1 *}$ Universidade Estadual do Centro-Oeste, Departamento de Engenharia Florestal, Irati, Paraná, Brasil - faretslaff@ gmail.com; \\ afonso.figueiredo@pq.cnpq.br \\ ${ }^{2}$ Universidade Federal do Paraná, Departamento de Ciências Florestais, Curitiba, Paraná, Brasil - samachado@ufpr.br; jarce@ufpr.br \\ ${ }^{3}$ Universidade Federal do Paraná, Programa de Pós-Graduação em Engenharia Florestal, Curitiba, Paraná, Brasil - \\ eldemarjaskiu@gmail.com
}

Recebido para publicação: 20/10/2014 - Aceito para publicação: 19/04/2016

\begin{abstract}
Resumo
Este trabalho teve como objetivo desenvolver um Diagrama de Manejo da Densidade (DMD) para definir momentos ótimos para aplicação de desbastes e corte final em povoamentos de Pinus taeda L., no estado do Paraná. A base de dados utilizada no estudo é oriunda de plantios de Pinus taeda estabelecidos no estado do Paraná, nas regiões Centro-Oeste, Sul e Campos Gerais, além de dados coletados na Floresta Nacional de Irati (FLONA de Irati) e em um experimento com diferentes espaçamentos existente no Campus da UNICENTRO (Universidade Estadual do Centro-Oeste), no município de Irati. Foram utilizadas 1.023 parcelas sem desbastes, com idades variando de 7 a 44 anos. Foi ajustado o modelo de densidade de Reineke (1933) para obter o Índice de Densidade de Reineke (IDR), construindo-se então, o DMD similarmente à metodologia proposta por McCarter e Long (1986) e Dean e Baldwin (1993), o qual tem como componentes básicos o IDR e um sistema de equações que relacionam o diâmetro médio quadrático $(d g)$ e o volume do povoamento $(V)$ com a densidade $(N)$, altura dominante $\left(h_{\text {dom }}\right)$ e o diâmetro médio quadrático. O sistema de equações ajustado para a construção do DMD apresentou estatísticas adequadas de ajuste e precisão, possibilitando a definição das densidades iniciais para atingir um dado diâmetro de tamanho desejado no corte final, bem como conhecer as idades em que devem ser realizadas as intervenções e a prognose da produção de cada colheita.

Palavras-chave: Autodesbaste; desbastes; manejo intensivo.
\end{abstract}

\begin{abstract}
Stand Density Management Diagram (SDMD) for Pinus taeda in the State of Parana. This work aimed to develop a stand density management diagram (SDMD) to define the best thinning schedule on Pinus taeda L. stands. The database used in the study came from Pinus taeda plantations established in state of Paraná, Brazil, in the Midwest, South and Campos Gerais regions, besides data collected from the National Forest of Irati and from an experiment with different spacings located on the campus of the State University of Midwest, in municipality of Irati. We used 1,023 plots without thinnings, with ages ranging from 7 to 44 years. Reineke density model (1933) was adjusted to obtain the Reineke Density Index (RDI), constructing then the DMD similarly to the methodology proposed by McCarter and Long (1986) and Dean and Baldwin (1993), which has as basic components, the IDR and a system of equations that relates the quadratic mean diameter $(d g)$ and the stand volume $(V)$ with the density $(N)$, dominant height $\left(h_{d o m}\right)$ and the quadratic mean diameter. The system of equations fitted for the construction of the DMD presented appropriate statistical adjustment and accuracy, allowing the definition of the initial densities to achieve a given diameter of the desired size in the clearcutting, as well as knowing the ages in which interventions should be carried out and prognosis of production of each harvest.
\end{abstract}

Keywords: Self-thinning; thinning; intensive management.

\section{INTRODUÇÃO}

É reconhecida a importância dos recursos advindos de florestas para atender às necessidades humanas. A madeira tem sido usada por séculos para construção de moradias, proteção, como combustível e matéria-prima. O uso desenfreado dos recursos florestais naturais levou a uma redução drástica desse recurso natural e assim, houve a necessidade de plantios florestais, com espécies de rápido crescimento. No Brasil, os plantios foram iniciados pela lei dos incentivos fiscais a partir de 1966 principalmente com os gêneros Pinus e Eucalyptus.

Muitos dos plantios florestais no Brasil foram conduzidos de maneira empírica, resultando em madeira de má qualidade, perda de madeira por mortalidade e ataque de pragas em povoamentos estressados (em competição). No entanto, a produção de madeira com boas características depende do uso de práticas

FLORESTA, Curitiba, PR, v. 46, n. 2, p. 185 - 195, abr. / jun. 2016.

Retslaff, F. A. de S. et al.

ISSN eletrônico 1982-4688

DOI: $10.5380 /$ rf.v46i2.38301 
silviculturais e do manejo adequado, os quais necessitam do conhecimento sobre a dinâmica de crescimento e a interação entre indivíduos no povoamento (LIMA et al., 2013).

A correta regulação da densidade é a chave para uma boa condução do povoamento em conformidade com os objetivos planejados, possibilitando a predição das condições futuras do povoamento. As técnicas de intervenções definidas pelo manejo intensivo têm por finalidade a obtenção de uma máxima produtividade e de um produto final com qualidade.

A condução da floresta para a obtenção de madeira para usos múltiplos, ou seja, madeira para celulose e papel, produção de móveis, construção civil, laminação, compensados, chapas de fibras, placas de OSB, entre outros, gera renda ao longo da rotação e agrega valores à matéria prima produzida. $\mathrm{O}$ chamado manejo intensivo busca otimizar o uso da floresta por meio de técnicas de intervenções ao longo do ciclo da mesma, produzindo fluxos de caixa intermediários e agregando maior valor à matéria prima produzida, principalmente aquela destinada ao corte final.

Segundo Dean e Baldwin (1993) os desbastes podem aumentar a produção total do povoamento com a utilização de árvores que de outra forma morreriam e, por meio da manutenção de um crescimento rápido das árvores individuais, minimizando a competição. No entanto, os desbastes devem ser programados corretamente para que os benefícios possam ser maximizados.

Ao longo dos anos, os pesquisadores vêm buscando desenvolver ferramentas baseadas em modelos matemáticos, incorporando aos mesmos conceitos biológicos, como a competição interespecífica, baseada em algumas das inúmeras relações de densidade e tamanho, como por exemplo, o número de árvores e o diâmetro médio quadrático, entre outras. Com a utilização de relações dendrométricas, torna-se possível construir importantes ferramentas para a condução de povoamentos florestais (SCHNEIDER et al., 2009; SELLE et al., 2010).

Como uma dessas ferramentas, podem-se citar os diagramas de manejo da densidade (DMDs). Estes diagramas são utilizados no planejamento de intervenções silviculturais, de maneira fácil e rápida, garantindo uma ideal ocupação do sítio, mediante um controle adequado dos níveis de competição, evitando perda de indivíduos pela mortalidade (autodesbaste) de acordo com os objetivos de produção propostos inicialmente (SELLE et al., 2010). Muito embora o cerne dessa modelagem do crescimento e da produção tenha se originado em uma proposta de Reineke (1933) há quase um século, os diagramas de densidade, nas duas últimas décadas, têm sido bastante pesquisados e aprimorados, notadamente em países europeus e nos Estados Unidos. Desta forma, têm sido usados nesses países como um instrumento para definir momentos ótimos de desbaste e facultativamente a poda.

Historicamente, DMDs foram desenvolvidos inicialmente por cientistas japoneses no início da década de 1960 empregando equações recíprocas de competição-densidade, efeito da produção-densidade e a regra do autodesbaste. Dentre esses cientistas, estão Ando (1962) e Tadaki (1963), que trabalharam com Pinus densiflora Siebold e Zucc e Cryptomeria japonica D. Don., respectivamente. Durante as décadas de 1970 e 1980 várias modificações ao método de modelagem original foram propostas, incluindo a reposição das equações recíprocas com as funções de densidade empíricas (baseadas no volume), empregando diferentes índices de densidade relativos e tamanhos variados, e a incorporação das teorias da produção florestal. Entre o início e a metade da década de 1990, DMDs foram construídos para várias espécies, empregando-se os métodos de modelagem mais recentes, incluindo o desenvolvimento de DMDs para povoamentos com espécies mistas (NEWTON, 1997).

Os diagramas de manejo da densidade são modelos gráficos simples da dinâmica de povoamentos de mesma idade. Eles refletem as relações fundamentais envolvendo tamanho, densidade, competição, ocupação do local e autodesbaste. São utilizados para determinar qual densidade depois do desbaste, resultará no tipo de povoamento desejado para a próxima intervenção. Os DMDs são também utilizados para exibir e avaliar densidades alternativas para regimes de manejo de acordo com os objetivos pretendidos, auxiliando na tomada de decisões. Segundo Cabanillas et al. (2009) os DMDs são ferramentas silviculturais de grande versatilidade e simplicidade para planejar programas de desbastes, segundo suas características e os objetivos de produção de madeira a serem obtidos no momento do corte. São ainda, especialmente interessantes, quando se dispõe de curvas de sítio para o local, a partir das quais, é possível conhecer outras variáveis, como a idade e a altura dominante (CABANILLAS et al., 2009; DEAN; BALDWIN, 1993).

Os DMDs são representados basicamente pelo número de árvores por hectare que deve ser mantido em um povoamento para se atingir o diâmetro médio quadrático igual a $25,4 \mathrm{~cm}$ (equivalente a 10 polegadas no sistema inglês). Assim, para um dado valor de diâmetro médio quadrático, existe um número máximo de árvores por unidade de área, independentemente da idade e da qualidade do sítio, em que as árvores a partir deste ponto entram em alto grau de competição, iniciando o autodesbaste (HUSCH et al., 1982).

Entre as várias alternativas existentes para se conduzir um povoamento florestal, deveria ser escolhida aquela que permita um máximo aproveitamento dos recursos e do local onde está implantado. Esse aproveitamento otimizado pode ser conseguido com uma ampla variedade de densidades, ou seja, uma faixa ótima de manejo, que varia entre um limite superior e outro inferior (CABANILLAS et al., 2009). O limite superior corresponde ao nível a partir do qual acontece mortalidade natural por concorrência. O limite inferior se 
estabelece quando todos os recursos do local são utilizados pelo povoamento, de modo que cada um dos indivíduos assimila tudo o que a sua condição genética e idade permitem. É necessário, então, decidir quando os indivíduos desenvolvem a sua máxima capacidade de crescimento. Abaixo desse limite perde-se capacidade produtiva (MADRIGAL, 1994; BARRIO-ANTA; GONZÁLEZ, 2005). Deste modo, os DMDs permitem que o silvicultor desenvolva rapidamente programas de desbastes para uma grande variedade e qualidades de sítio, com base nos objetivos do manejo (DEAN; BALDWIN, 1993).

Os diagramas de densidades aprimorados nas últimas décadas, praticamente não têm sido aplicados no Brasil, onde até pouco tempo atrás, predominavam os grandes empreendimentos florestais. Todavia, atualmente, muitos pequenos e médios proprietários também têm se dedicado às atividades florestais. Assim sendo, os modernos DMDs podem se constituir em uma ferramenta de grande utilidade para grandes e, sobretudo, para os pequenos e médios produtores florestais, considerando as facilidades de aplicação desses diagramas. Nesse caso, vislumbra-se um grande potencial para os Engenheiros Florestais autônomos que podem sugerir, a baixo custo, o manejo que deve ser aplicado nos pequenos e médios plantios florestais.

De acordo com o exposto, se o fundamento dado pelo diagrama de densidade for incorporado às técnicas de manejo intensivo, torna-se possível atingir florestas com ciclos regulares e balanceados, capazes de fornecer, ao longo de sua rotação, uma variedade maior de produtos com alta qualidade, gerando renda intermediária e agregando valor ao produto final. Dessa maneira, o presente trabalho teve como objetivo desenvolver um Diagrama de Manejo da Densidade (DMD) para definir momentos ótimos de intervenções (desbastes) para Pinus taeda L. no estado do Paraná.

\section{MATERIAL E MÉTODOS}

\section{Área de estudo e fonte de dados}

A base de dados utilizada no estudo é oriunda de plantios de Pinus taeda localizados no estado do Paraná nas regiões Centro-Oeste, Sul e Campos Gerais, além de dados coletados na Floresta Nacional de Irati (FLONA de Irati) e em um experimento com diferentes espaçamentos existente no Campus da UNICENTRO (Universidade Estadual do Centro-Oeste), em Irati.

Para o ajuste do modelo de densidade de Reineke (1), assim como para o sistema de equações baseado nos modelos (3) e (4), foram utilizados dados provenientes de 1.023 parcelas sem desbastes, com idades variando de 7 a 44 anos. Uma melhor descrição da área de estudo, assim como da base de dados pode ser encontrada em Retslaff (2014).

\section{Construção do Diagrama de Manejo da Densidade (DMD)}

O cerne dos Diagramas de Manejo da Densidade (DMDs) são os Índices de Densidade, e entre eles o de maior uso é o Índice de Densidade de Reineke (IDR). No DMD é possível obter diretamente variáveis como o diâmetro médio quadrático $(d g)$ e o número de árvores por hectare $(N)$. Alguns diagramas mais recentes possuem linhas que representam a altura dominante e também linhas que determinam o volume por hectare $(V)$.

Para a construção do DMD, primeiro foi necessário ajustar o modelo (1) para obter o IDR (2):

$$
\begin{aligned}
\log (N) & =\beta_{0}+\beta_{1} \log (d g) \\
I D R & =N\left(\frac{d g}{25,4}\right)^{-\beta_{1}}
\end{aligned}
$$

em que: $\mathrm{dg}=$ diâmetro médio quadrático $(\mathrm{cm}) ; N=$ número de árvores por hectare; $\log =\operatorname{logaritmo}$ na base $10 ; \beta_{i}$ $(\mathrm{i}=0-1)=$ coeficientes de regressão.

O DMD foi construído similarmente à metodologia proposta por McCarter e Long (1986) e Dean e Baldwin (1993), os quais utilizaram os modelos (3) e (4) para plotar as linhas de altura dominante no DMD (Figura 1).

Os componentes básicos do DMD desenvolvido envolvem o IDR (2) e as duas equações (3 e 4) que relacionam o diâmetro médio quadrático $(d g)$ e o volume do povoamento $(V)$ com a densidade $(N)$, altura dominante $\left(h_{d o m}\right)$ e o diâmetro médio quadrático (VALBUENA et al., 2008; DEAN; BALDWIN, 1993):

$$
\begin{gathered}
d g=\beta_{0} V^{\beta_{1}} N^{\beta_{2}} \\
V=\beta_{3} d g^{\beta_{4}} h_{d o m}^{\beta_{5}} N
\end{gathered}
$$

em que: $\mathrm{dg}=$ diâmetro médio quadrático $(\mathrm{cm}) ; h_{\text {dom }}=$ altura dominante $(\mathrm{m}) ; N=$ número de árvores por hectare; $V=$ volume total $\left(\mathrm{m}^{3} / \mathrm{ha}\right) ; \beta_{i}(\mathrm{i}=0$ a 5$)=$ coeficientes de regressão. 
As equações (3) e (4) são usadas para plotar as linhas de altura dominante no DMD (Figura 1) e foram ajustadas, simultaneamente, utilizando-se o FIML (Full Information Maximum Likelihood), mediante o procedimento (PROC) MODEL do programa SAS (VALBUENA et al., 2008). Para avaliação dos modelos ajustados foi utilizado o coeficiente de determinação ajustado $\left(R_{a j}^{2}\right)$ e o erro padrão de estimativa $\left(S_{y x}\right)$.

Para plotar as linhas de alturas dominantes no gráfico foi necessário isolar a variável $d g$, manter fixa a variável $h_{d o m}$ e variar o $N$. Maiores detalhes para plotar as linhas de alturas dominantes estão em Retslaff (2014).

Utilizando-se o modelo de densidade de Reineke (1) foi determinada a linha de máxima densidade de árvores por hectare pelo diâmetro médio (SDI-100-stand density index - índice de densidade do povoamento Figura 1) e as linhas que limitam as zonas das diferentes fases de concorrência durante o desenvolvimento do povoamento. A faixa ótima de manejo (linhas pontilhadas na Figura 1) para Pinus taeda utilizada por Dean e Baldwin (1993) e definida por Harrington (1997), nos Estados Unidos da América, situa-se entre 30 e $45 \%$ do máximo SDI. Esses percentuais foram também adotados nesta pesquisa. As linhas limites são determinadas algebricamente a partir da linha de máxima densidade.

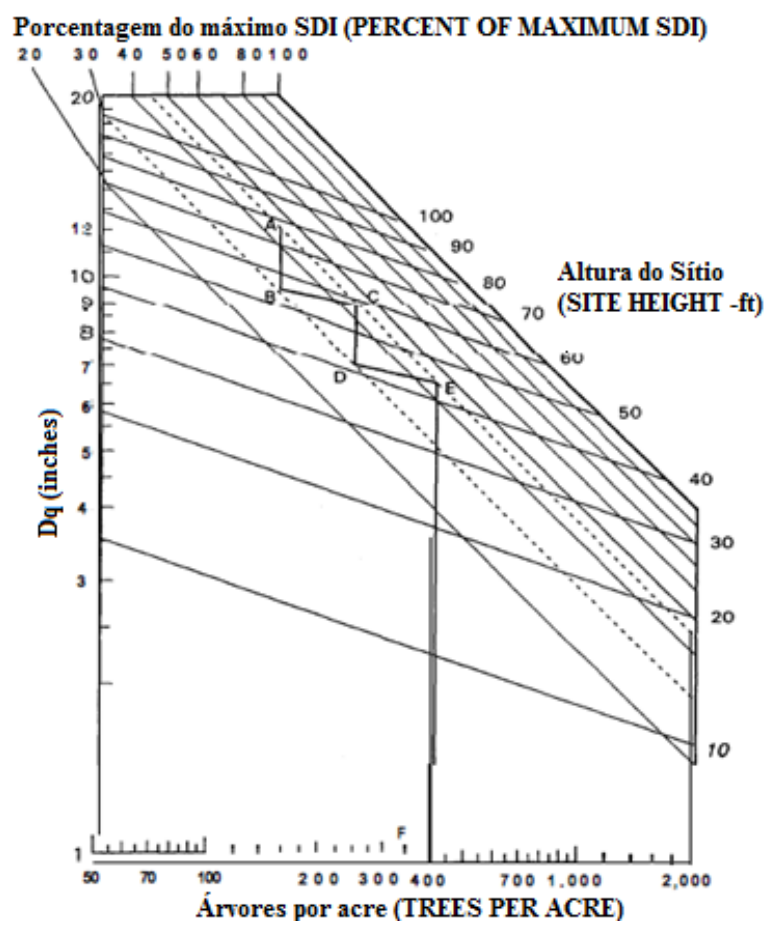

Figura 1. Diagrama de Manejo da Densidade para Pinus taeda, mostrando um exemplo de sequência de desbastes. SDI: Stand Density Index (Índice de densidade do povoamento); Dq: diâmetro dg; ft: pés; A a D: sequência de desbastes; E: corte final. Fonte: Dean e Baldwin (1993) - traduzido por Retslaff (2014).

Figure 1. Stand density management diagrams for Pinus taeda, showing an example of a sequence of thinnings; SDI: Stand Density Index, Dq: quadratic mean diameter; ft: feet; A a D: sequence of thinnings; E: final harvest. Source: Dean and Baldwin (1993) - translated by Retslaff (2014).

Na prática, a linha de máxima densidade (na Figura 1 representada pelo SDI $=100$ ), é uma projeção da linha descrita pelo modelo (1) que expressa a média dos pontos. Isto é realizado incrementando-se o valor do intercepto " $\beta_{0}$ " do modelo ajustado (1), elevando a linha para o limite superior, até que não ocorram mais pontos acima, em um diâmetro padrão de $25,4 \mathrm{~cm}$ (equivalente a 10 polegadas no sistema Inglês), ou seja, essa elevação da linha média ajustada pelo modelo (1) é realizada isolando-se o $\beta_{0}$ no modelo e definindo $d g$ como sendo o diâmetro padrão de $25,4 \mathrm{~cm}$ e o $N$ como sendo o IDR (5), conforme segue:

$$
\beta_{0}=\log (I D R)-\beta_{1} \log (25,4)
$$

\section{Uso do Diagrama de Manejo para planejamento de desbastes}

Para estimar o crescimento e a produção por meio do DMD e simular desbastes foi utilizada uma metodologia similar à empregada por Dean e Baldwin (1993). Primeiramente é necessário dispor do número de 
árvores por hectare $(N)$. A partir do $N$, traça-se uma linha reta perpendicular ao eixo " $X$ ” $(N)$ até atingir a segunda linha pontilhada do ponto "E" (Figura 1). Nesse ponto, localiza-se no eixo " $Y$ " $(d g)$ o diâmetro $d g$ correspondente. Para encontrar a altura dominante correspondente ao ponto "E", deve-se traçar uma linha paralela às linhas de alturas dominantes no gráfico. Assim, as linhas de alturas dominantes (dominant heights) desenhadas no diagrama (Figura 1) em conjunto com curvas de índice de sítio disponíveis para o local, permitem determinar as idades dos desbastes e do corte final (pontos "E", "C" e "A"), conhecendo-se o índice de sítio ao qual o povoamento pertence.

Resumindo, no DMD é possível obter as variáveis $h_{d o m}, d g, N$ e $G$. Com o $h_{d o m}$ e o índice de sítio $(S)$ do povoamento, determinam-se as idades dos desbastes (E e C) e corte final (A). Nesta pesquisa foram utilizadas as curvas de sítio desenvolvidas por Retslaff (2014), assim como o modelo de sobrevivência até a idade do primeiro desbaste. Considerou-se que, depois dos desbastes não ocorre mortalidade, desde que os desbastes sejam realizados, mantendo-se o povoamento dentro da "faixa ótima de manejo", indicada pelo Diagrama de Manejo da Densidade.

\section{Desbastes}

Quando o diâmetro $d g$ de um povoamento atinge o ponto "E”, “C” ou o ponto "A" (Figura 1) é o momento de intervir no povoamento, seja com desbastes (pontos "E" e "C") ou com o corte final (ponto "A"). Conduzir o povoamento com crescimento além da linha pontilhada superior, que representa o início da mortalidade ou autodesbaste, implica em perder árvores devido à mortalidade causada por competição.

Se o interesse é manejar o povoamento para obter múltiplos produtos, deve-se intervir com desbastes para manter o povoamento dentro da faixa ótima de manejo (faixa entre as linhas pontilhadas). Para conhecer a localização dos pontos B e D na figura 1 é necessário conhecer a mudança do diâmetro $d g$ depois dos desbastes. Em um exemplo de programação de desbates com o uso de um DMD, Dean e Baldwin (1993) comentaram que análises com o programa de crescimento e produção COMPUTE_P-LOB para Pinus taeda (FERGUSON; BALDWIN, 1987) indicavam um incremento (mudança) em torno de 1,07 (ou 7\%) no diâmetro $d g$ após os desbastes. Assim, os segmentos $\overline{D E}$ e $\overline{B C}$ (Figura 1) foram desenhados por esses autores de modo que a razão entre o diâmetro $d g$ depois dos desbastes e o diâmetro $d g$ antes dos desbastes fosse igual a 1,07.

Seguindo essa metodologia, foram calculados os diâmetros $d g$ antes e depois dos desbastes com base em parcelas medidas no inventário pré-corte da empresa REMASA, em que as árvores selecionadas para serem colhidas no desbaste foram marcadas com um código. Os desbastes realizados eram do tipo misto (seletivo sistemático), retirando-se a $5^{\text {a }}$ linha (sistemático) e no seletivo eram colhidas árvores suprimidas ou defeituosas (finas, tortas, bifurcadas). Foram utilizados dados de 40 parcelas para o primeiro desbaste e 29 parcelas para o segundo desbaste (D2).

A razão entre os valores depois e antes $\left(\frac{d g_{\text {depois }}}{d g_{\text {antes }}}\right)$ foi calculada para o primeiro e segundo desbastes. Conhecendo essa mudança, foram determinados os pontos " $\mathrm{B}$ " e " $\mathrm{D}$ " no diagrama (Figura 1). Localizando esses pontos tem-se o número de árvores após os desbastes (correspondente a 241 e 150 árvores/acre na Figura 1).

\section{RESULTADOS E DISCUSSÃO}

\section{Modelos para construção do diagrama}

Na tabela 1 estão apresentados os resultados dos ajustes dos modelos (1), (3) e (4) utilizados na construção do Diagrama de Manejo da Densidade (DMD).

Tabela 1. Coeficientes e estatísticas dos modelos ajustados para construção do Diagrama de Manejo da Densidade.

Table 1. Coefficients and statistics of the adjusted models for the construction of the Density Management Diagram.

\begin{tabular}{|c|c|c|c|c|c|c|}
\hline Autor/Fonte/Modelo & Coeficientes & $\mathbf{R}^{2} \mathbf{a d j}$ & $\mathbf{S}_{\mathrm{yx}}$ & $\mathrm{S}_{\mathrm{yx}} \%$ & $\mathbf{n}$ & $\mathbf{N}^{\circ}$ \\
\hline $\begin{array}{l}\text { Reineke }(1933) \\
\log (N)=\beta_{0}+\beta_{1} \log (d g)\end{array}$ & $\begin{array}{l}\beta_{0}=5,50923 \\
\beta_{1}=-1,76714\end{array}$ & 0,8232 & $\begin{array}{l}366,19 \\
\text { (N/ha) }\end{array}$ & 26,31 & 1.023 & (1) \\
\hline $\begin{array}{l}\text { Dean e Baldwin (1993) } \\
d g=\beta_{0} V^{\beta_{1}} N^{\beta_{2}}\end{array}$ & $\begin{array}{l}\beta_{0}=122,29080 \\
\beta_{1}=0,19169 \\
\beta_{2}=-0,39874\end{array}$ & 0,9457 & $1,25(\mathrm{~cm})$ & 4,90 & 1.023 & (3) \\
\hline $\begin{array}{l}\text { Dean e Baldwin (1993) } \\
V=\beta_{3} d g^{\beta_{4}} h_{\text {dom }}^{\beta 5} N\end{array}$ & $\begin{array}{l}\beta_{3}=2,40 \mathrm{E}-5 \\
\beta_{4}=2,08939 \\
\beta_{5}=1,01926\end{array}$ & 0,9212 & $56,08\left(\mathrm{~m}^{3} / \mathrm{ha}\right)$ & 11,04 & 1.023 & (4) \\
\hline
\end{tabular}

em que: $d g=$ diâmetro médio quadrático $(\mathrm{cm}) ; h_{d o m}=$ altura dominante $(\mathrm{m}) ; N=$ número de árvores por hectare; $V=$ volume total $\left(\mathrm{m}^{3} / \mathrm{ha}\right)$; $R^{2}$ adj $=$ Coeficiente de determinação ajustado; $S_{y x}=$ Erro padrão de estimativa; $\beta_{i s}=$ coeficientes estimados; $\mathrm{n}=$ número de observações; $\mathrm{N}^{\circ}=$ número do modelo. 
O valor para o coeficiente $\beta_{1}$ estimado para Pinus taeda foi diferente do coeficiente angular sugerido por Reineke (1933) $\left(\beta_{1}=-1,605\right)$, quando testou o modelo para várias espécies, além de ser diferente da lei da potência $-3 / 2$ de autodesbaste. Schneider (2008) trabalhou com dados de $P$. taeda em Santa Catarina e obteve para o coeficiente angular o valor de $-1,4482 \mathrm{com} R_{a d j}^{2}$ de 0,88 e $S_{y x}$ de $1,25 \%$. A diferença na qualidade do ajuste e precisão em relação à presente pesquisa pode ser explicada pela grande variabilidade dos dados que provêm de diferentes regiões.

Chauchard e Olalde (2004) estimaram para Pinus radiata no País Vasco o valor de $-1,89909$ para o coeficiente angular do modelo de Reineke (1933). Saunders e Puettman (2000) obtiveram para Picea glauca, em Lake States, EUA, um coeficiente angular de -0,473 para o modelo de Reineke. Del Río et al. (2001) citaram que essa inclinação (coeficiente angular) ou taxa de autodesbaste foi calculada para diferentes espécies em pesquisas posteriores, obtendo-se inclinações diferentes com a espécie, e às vezes com a idade, com valores entre $-2,33$ e 1,54. De acordo com Schneider (2008) isso indica que o coeficiente angular do modelo de Reineke (1933) é diferente para cada espécie e não constante, o que, segundo esse autor, não corrobora com o que foi inicialmente formulado por Reineke em 1933.

Apesar de muitas pesquisas mostrarem que o valor do coeficiente angular do modelo de Reineke (1933) difere do postulado inicialmente pelo autor, vários pesquisadores, como por exemplo, McCarter e Long (1986); Dean e Baldwin (1993); Harrington (1997); e Valbuena et al. (2008), utilizaram o coeficiente de -1,605 (ou -1,6) para plotar o Índice de Densidade de Reineke nos DMD. No entanto, segundo Chauchard e Olalde (2004) uma consideração particularmente importante em relação à localização e inclinação da função que expressa a densidade máxima, está relacionada com a utilização posterior para a simulação de mortalidade natural e o planejamento da produção total de determinado regime silvicultural. Ainda de acordo com esses autores, se a linha de referência superior não tem relação com as reais máximas densidades médias, tem-se o risco de estar deixando crescer o povoamento até níveis populacionais muito altos ou ainda pior, inatingíveis, e com isto estar distorcendo a estimativa das densidades e da produção.

$\mathrm{Na}$ figura 2 está apresentada a curva média estimada pelo modelo (1) passando pelos valores observados. O gráfico foi construído em escala logarítmica (duplo log) com uma inclinação de -1,7671, obtida neste trabalho.

O sistema de equações apresentou boas estatísticas de ajuste e precisão (Tabela 1). O ajuste desse sistema foi utilizado por Dean e Baldwin (1993) para plotar as linhas de altura dominante de Pinus taeda nos Estados Unidos, no DMD utilizado para simular programas de desbastes. Esses autores obtiveram um Erro Padrão (EP) de 1,37 cm para o diâmetro e EP igual a 36,664 m³/ha para o volume.

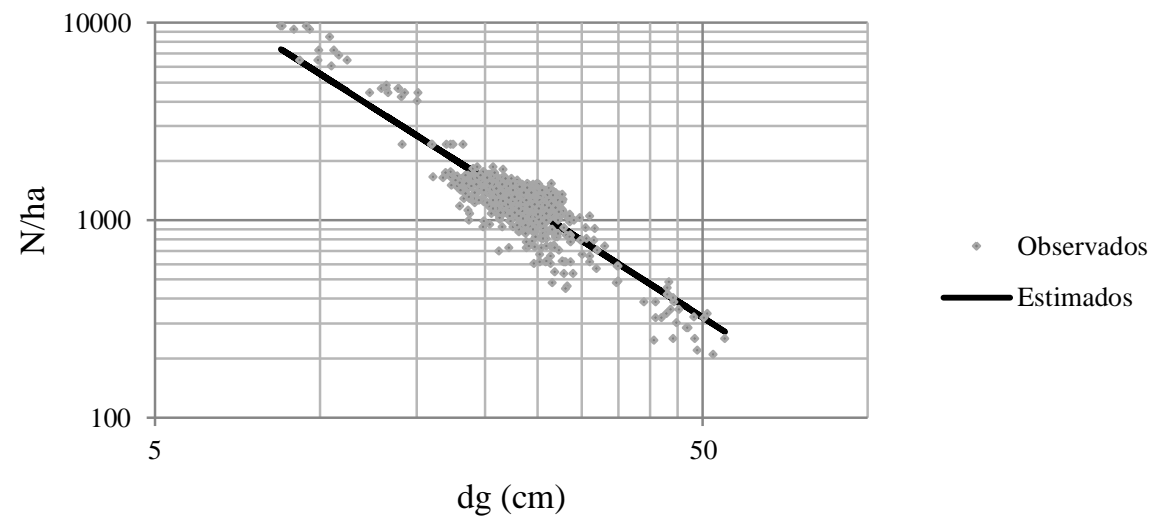

Figura 2. Distribuição gráfica dos valores observados e a linha média estimada (em escala duplo-log) pelo modelo de Reineke (1933) para Pinus taeda no estado do Paraná.

Figure 2. Graphical distribution of observed and estimated average line (double-log scale) for the Reineke model (1933) for Pinus taeda in State of Parana.

Dean e Baldwin (1993) comentaram que as linhas de alturas dominantes no DMD desenvolvido para $P$. taeda diferem substancialmente das linhas do DMD para $P$. contorta var. latiofolia Engelm. de McCarter e Long (1986) e do DMD para $P$. elliiottii de Dean e Jokela (1992). Segundo ainda esses autores, em $P$. contorta e $P$. elliiottii somente em densidades extremamente altas a relação entre altura e diâmetro é afetada significativamente. Em P. taeda, entretanto, o diâmetro associado a uma determinada altura diminui rapidamente com o aumento da densidade do povoamento. 
Na figura 3 está apresentado o DMD construído para Pinus taeda para plantios estabelecidos no estado do Paraná, em que estão apresentadas as linhas de máxima densidade e a faixa ótima de manejo, entre 30 e $45 \%$ da máxima densidade. As linhas de altura dominante foram plotadas em uma amplitude de $3 \mathrm{~m}$, variando de 10 a $37 \mathrm{~m}$, apenas para ilustração. Nos exemplos apresentados na figura 4, essas linhas foram geradas apenas para as alturas dominantes estimadas em cada simulação, com intuito de facilitar a visualização.

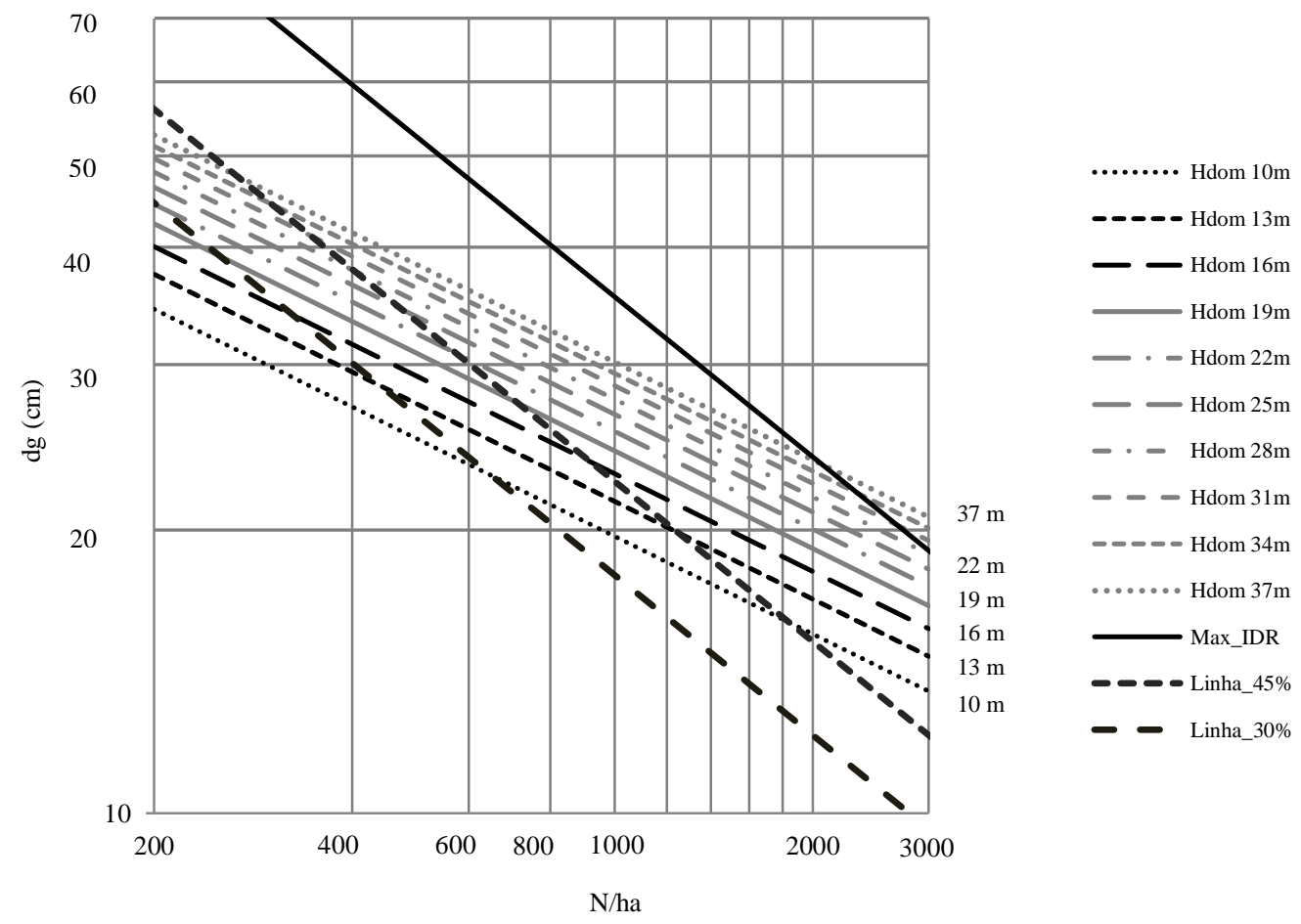

Figura 3. Diagrama de Manejo da Densidade para plantios de Pinus taeda no estado do Paraná.

Figure 3. Stand Density Management Diagrams for plantations of Pinus taeda in State of Parana.

\section{Desbastes}

De acordo com o que foi mencionado na metodologia, para conhecer o diâmetro $d g$ depois dos desbastes, é necessário o entendimento da mudança que ocorre nesse diâmetro após as intervenções. Como os desbastes são mistos, o diâmetro $d g$ deve aumentar após o desbaste, uma vez que, além de serem colhidas árvores em todas as classes (desbaste sistemático), as árvores mais finas geralmente são as escolhidas para serem cortadas, além das árvores quebradas, bifurcadas, etc. (desbaste seletivo). Para conhecer essa mudança, foi calculado o diâmetro $d g$ antes e depois dos desbastes e encontrada a mudança ocorrida nessa variável.

Seguindo a metodologia, foram calculados os diâmetros $d g$ antes e depois dos desbastes. A razão entre os valores depois e antes $\left(\frac{d g_{\text {depois }}}{d g_{\text {antes }}}\right)$ foi determinada para o primeiro e segundo desbastes, resultando em uma média de 1,0719 e 1,0689, respectivamente. Esses valores são muito próximos aos encontrados por Dean e Baldwin (1993) para Pinus taeda nos Estados Unidos (1,07).

\section{Exemplo de planejamento de desbastes com o DMD}

Na sequência são apresentados dois exemplos de uso do DMD para o planejamento de desbastes, tendo como objetivo manter o povoamento dentro da faixa ótima de manejo, a fim de evitar a mortalidade de árvores por competição.

No primeiro exemplo, a simulação teve início com uma densidade inicial de 1.600 árvores/ha. Fazendo uso do modelo de sobrevivência $N_{s}=\left\{N_{p} \cdot\left[\exp \left(I\left(0,0654487-0,00989517 \ln \left(N_{p}\right)\right)\right]\right\}\right.$, ajustado por Retslaff (2014) (em que: $N_{s}=$ número de árvores da medição realizada na idade $I ; N_{p}=$ número de árvores/ha plantadas; $I=$ idade (anos); exp = exponencial), é possível estimar o número de árvores sobreviventes na idade do primeiro desbaste.

A idade do primeiro desbaste é encontrada localizando a altura dominante correspondente ao número de árvores sobrevivente $\left(N_{S}\right)$ no DMD. Com base nessa altura dominante e conhecendo o índice de sítio $(S)$ ao qual o 
povoamento pertence, encontra-se a idade. Como a idade é encontrada com base na altura dominante e esta variável depende do $N_{s}$ para ser estimada, é necessário fazer uso de algum processo iterativo para encontrar essas variáveis. Neste exemplo foi utilizado o Microsoft Excel. Esse problema pode ser evitado se o número de árvores sobreviventes for obtido como uma porcentagem do número de árvores plantadas ou, como apresentado em alguns exemplos na literatura, não se leva em consideração a mortalidade inicial de estabelecimento do povoamento, e o número de árvores sobreviventes antes do primeiro desbaste é igual ao número de árvores plantadas.

$\mathrm{Na}$ tabela 2 estão apresentados os resultados para o primeiro exemplo, onde se observa que os valores estimados para cada um dos três índices de sítio são muito próximos para uma mesma densidade inicial (no exemplo, 1.600 árvores/ha), todavia, como esperado, a idade em que devem ocorrer as intervenções é diferente. Em sítios bons a competição inicia-se antes com o maior crescimento, atingindo mais cedo a linha de densidade de $45 \%$, estabelecida como sendo o limite para o início do autodesbaste, ou seja, se não forem feitas intervenções (desbastes ou o corte raso) para que o povoamento continue crescendo, algumas árvores morrerão devido à competição.

Tabela 2. Atributos do povoamento antes de cada intervenção.

Table 2. Stand attributes before each intervention.

\section{Exemplo 1: Densidade inicial $=1.600$ árvores $/$ ha}

\begin{tabular}{|c|c|c|c|c|c|}
\hline \multicolumn{6}{|c|}{ Antes do primeiro desbaste } \\
\hline$S(\mathrm{~m})$ & Ns/ha & Idade (anos) & $d g(\mathrm{~cm})$ & $G\left(\mathrm{~m}^{2} / \mathrm{ha}\right)$ & $h_{d o m}(\mathrm{~m})$ \\
\hline 18 & 1.486 & 9,8 & 18,0 & 37,888 & 11,6 \\
\hline 24 & 1.517 & 7,1 & 17,8 & 37,788 & 11,4 \\
\hline 30 & 1.533 & 5,7 & 17,7 & 37,735 & 11,3 \\
\hline \multicolumn{6}{|c|}{ Antes do segundo desbaste } \\
\hline$S(\mathrm{~m})$ & $\mathrm{Nr} / \mathrm{ha}$ & Idade (anos) & $d g(\mathrm{~cm})$ & $G\left(\mathrm{~m}^{2} / \mathrm{ha}\right)$ & $h_{d o m}(\mathrm{~m})$ \\
\hline 18 & 876 & 15,6 & 24,3 & 40,619 & 16,5 \\
\hline 24 & 894 & 10,4 & 24,0 & 40,512 & 16,3 \\
\hline 30 & 904 & 8,0 & 23,9 & 40,455 & 16,2 \\
\hline \multicolumn{6}{|c|}{ Antes do corte raso } \\
\hline$S(\mathrm{~m})$ & $\mathrm{Nr} / \mathrm{ha}$ & Idade (anos) & $d g(\mathrm{~cm})$ & $G\left(\mathrm{~m}^{2} / \mathrm{ha}\right)$ & $h_{d o m}(\mathrm{~m})$ \\
\hline 18 & 519 & 42,3 & 32,7 & 43,518 & 23,5 \\
\hline 24 & 530 & 17,0 & 32,3 & 43,403 & 23,2 \\
\hline 30 & 536 & 12,1 & 32,1 & 43,343 & 23,1 \\
\hline \multicolumn{6}{|c|}{ Exemplo 2: Densidade inicial = 1.074 árvores $/$ há } \\
\hline \multicolumn{6}{|c|}{ Antes do primeiro desbaste } \\
\hline$S(\mathrm{~m})$ & $N s / h a$ & Idade (anos) & $d g(\mathrm{~cm})$ & $G\left(\mathrm{~m}^{2} / \mathrm{ha}\right)$ & $h_{d o m}(\mathrm{~m})$ \\
\hline 24 & 1.039 & 9,3 & 22,1 & 39,720 & 14,7 \\
\hline 30 & 1.046 & 7,3 & 22,0 & 39,683 & 14,7 \\
\hline \multicolumn{6}{|c|}{ Antes do segundo desbaste } \\
\hline$S(\mathrm{~m})$ & $\mathrm{Nr} / \mathrm{ha}$ & Idade (anos) & $d g(\mathrm{~cm})$ & $G\left(\mathrm{~m}^{2} / \mathrm{ha}\right)$ & $h_{\text {dom }}(\mathrm{m})$ \\
\hline 24 & 612 & 14,5 & 29,8 & 42,583 & 21,1 \\
\hline 30 & 617 & 10,7 & 29,6 & 42,543 & 21,0 \\
\hline \multicolumn{6}{|c|}{ Antes do corte raso } \\
\hline$S(\mathrm{~m})$ & $\mathrm{Nr} / \mathrm{ha}$ & Idade (anos) & $d g(\mathrm{~cm})$ & $G\left(\mathrm{~m}^{2} / \mathrm{ha}\right)$ & $h_{d o m}(\mathrm{~m})$ \\
\hline 24 & 363 & 32,2 & 40,0 & 45,623 & 30,0 \\
\hline 30 & 366 & 17,9 & 39,8 & 45,580 & 29,9 \\
\hline
\end{tabular}

em que: $S$ = índice de Sítio na idade índice de 18 anos; $N s$ = número de árvores sobreviventes por hectare na idade do primeiro desbaste; $N r$

$=$ número de árvores remanescente após cada intervenção; $d g=$ diâmetro médio quadrático; $G=$ área basal; $h_{d o m}=$ altura dominante.

A idade do corte final para $S=18 \mathrm{~m}$ foi bastante elevada (Tabela 2), isso se deve ao fato de que a idade é calculada com base na equação de sítio, apenas isolando a variável idade $(I)$, deixando-a em função da altura dominante $\left(h_{d o m}\right)$ e do índice de sítio $(S)$. Nesta pesquisa foi usado o modelo biológico de Chapman-Richards, o qual, quando as alturas estão próximas ao seu valor assintótico, o valor da altura estimada tem mínima variabilidade de ano para ano. Assim, para atingir exatamente os 23,5 m no $S=18 \mathrm{~m}$, o povoamento levaria 42,3 anos. Analisando os valores gerados para as alturas dominantes com o modelo de Chapman-Richards, foi observado que por volta dos 34 anos as alturas dominantes já haviam atingido $94 \%$ do seu valor assintótico. Além disso, em comparações realizadas com o SisPinus (OLIVEIRA et al., 1991) a produção gerada aos 34 anos 
pelo DMD foi $454,3 \mathrm{~m}^{3} /$ ha e com o SisPinus foi $434,3 \mathrm{~m}^{3} /$ ha, resultando em uma diferença de $4,6 \%$. Deve ser ressaltado que para esse índice de sítio tal regime de manejo não é apropriado.

Na figura 4 (a) pode-se observar a sequência de desbastes no DMD. As linhas pontilhadas representam a faixa ótima de manejo e, quando o povoamento atinge a linha dos $45 \%$, ou seja, $45 \%$ da máxima densidade para o povoamento, é o momento de intervir na floresta para evitar mortes de árvores por competição, reduzindo a densidade até atingir novamente a linha dos $30 \%$. Se o povoamento ficar abaixo dessa linha, o potencial de produção do local estaria sendo subutilizado.

No segundo exemplo o diagrama foi utilizado para fazer simulações utilizando um diâmetro médio objetivo, ou seja, para atingir um diâmetro médio $d g$ objetivo no corte final, quantas árvores deveriam ser plantadas. Esse sistema é bastante utilizado nos países que fazem uso do DMD para o planejamento da produção. Seria como ir no sentido inverso no DMD. Neste exemplo, o DMD foi utilizado para planejar a implantação de povoamento com o objetivo de ter no corte final um diâmetro médio $d g$ de $40 \mathrm{~cm}$, chamado de diâmetro objetivo. A simulação foi realizada para dois índices de sítio, 24 e $30 \mathrm{~m}$ e os resultados dos atributos preditos para o povoamento podem ser observados na tabela 2. Para o índice de sítio de $18 \mathrm{~m}$, a altura dominante estimada no corte raso seria próxima aos valores obtidos para os demais sítios, no entanto, a altura dominante assintótica estimada pelo modelo de Chapman-Richards para esse sítio é $24,23 \mathrm{~m}$, ou seja, não há como o povoamento atingir a altura de $30 \mathrm{~m}$ no sítio de $18 \mathrm{~m}$.

Para atingir um diâmetro $d g$ de $40 \mathrm{~cm}$ deveriam ser plantadas 1.074 árvores/ha, resultando em um corte raso aos 32,2 anos para o sítio $24 \mathrm{~m}$ e 17,9 anos para o sítio $30 \mathrm{~m}$, simulando dois desbastes. A sequência de desbastes pode ser observada na figura 4 (b).
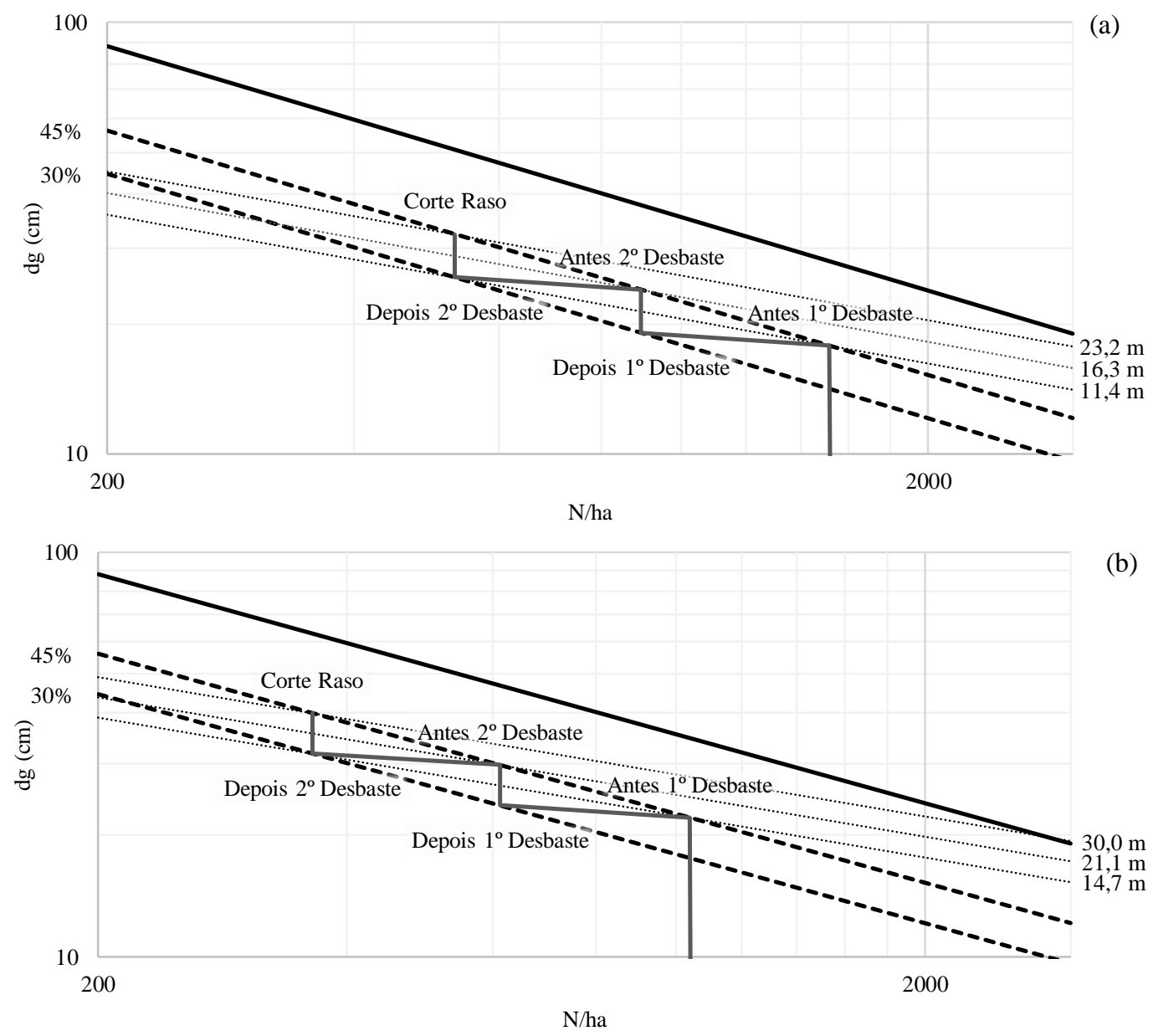

Figura 4. Sequência de desbastes para um povoamento de Pinus taeda. (a) densidade inicial de 1600 árvores/ha (b) densidade inicial de 1074 árvores/ha.

Figure 4. Thinning sequence for a Pinus taeda stand. (a) initial density of 1600 trees/ha (b) initial density of 1074 trees/ha. 


\section{CONCLUSÕES}

- O sistema de equações ajustado para a construção do Diagrama de Manejo da Densidade (DMD) apresentou estatísticas adequadas de ajuste e precisão, podendo ser utilizado como ferramenta para definir regimes de manejo para plantios de Pinus taeda, no estado do Paraná.

- O DMD construído indica que para atingir produções similares em um plantio com a densidade frequentemente usada no sul do Brasil (1.600 árvores/ha), as idades ótimas de desbastes em sítios bons ( $\mathrm{S}=$ $30 \mathrm{~m}$ ) ocorrem aos 6 e 8 anos, com corte final aos 12 anos, enquanto que para a mesma densidade em um sítio ruim $(\mathrm{S}=18 \mathrm{~m})$ essas idades ótimas ocorrem aos 10, 16 e 42 anos, respectivamente.

- O diagrama mostra também que para atingir um diâmetro objetivo de $40 \mathrm{~cm}$ em um povoamento com cerca de 1.000 árvores/ha seria necessária uma rotação de 32 anos para um sítio médio $(\mathrm{S}=24 \mathrm{~m})$ e 18 anos para um sítio bom $(\mathrm{S}=30 \mathrm{~m})$.

- O DMD construído possibilita a regulação de regimes de manejo definindo idades ótimas para a realização de desbastes e corte final, evitando-se o autodesbaste. Além disto, é possível determinar a densidade inicial para atingir um diâmetro objetivo.

\section{REFERÊNCIAS}

ANDO, T. Growth Analysis on the Natural Stands of Japanese Red Pine (Pinus densiflora Sieb. et. Zucc.). II. Analysis of Stand Density and Growth (In Japanese; English Summary). Government of Japan, Bulletin of the Government Forest Experiment Station (Tokyo, Japan) No. 147, 1 - 77. 1962.

BARRIO-ANTA, M.; GONZÁLEZ, J. G. Development of a stand density management diagram for even-aged pedunculate oak stands and its use in designing thinning schedules. Forestry, v. 78, n. 3, 2005.

CABANILlAS, A.; BARRIO ANTA, M.; ALBORECA, A. R.; NOTIVOL, E. P. Diagrama de manejo de densidad para masas naturales de pino carrasco (Pinus halepensis Mill.) en la depresión del Ebro. $\mathbf{5}^{\mathbf{0}}$ Congreso Forestal Español: Montes y sociedad: Saber qué hacer. Editores: S. E. C. F.- Junta de Castilla y León Ávila, 21 a 25 de septiembre de 2009. Sociedade Española de Ciencias Forestales. 13 p.

CHAUCHARD, L.; OLALDE, M. Diagrama de manejo de la densidad de masas de pino radiata en el País Vasco. Cuad. Soc. Esp. Cien. For. 18: 161 - 166 (2004).

DEAN, T. J.; JOKELA, E. J. A density management diagram for slash pine plantations in the lower Coastal Plain. Southern Journal of Applied Forestry. 16(4): 178 - 185. 1992.

DEAN, T. J.; BALDWIN JR., V. C. Using a Density-Management Diagram to Develop Thinning Schedules for Loblolly Pine Plantations. New Orleans, LA: U.S. Department of Agriculture, Forest Service, Southern Forest Experiment Station. 1993. 7 p.

DEL RÍO, M.; MONTEROB, G.; BRAVO, F. Analysis of diameter density relationships and self-thinning in non-thinned even-aged Scots pine stands. Forest Ecology and Management, 142 (2001) 79 - 87.

FERGUSON, R. B.; BALDWIN, V. C., Jr. Comprehensive outlook for managed pines using simulated treatment experiments-planted loblolly pine (COMPUTE-PLOB ): A user's guide. Res. Pap. SO-241. New Orleans, LA; U.S. Department of Agriculture, Forest Service, Southern Forest Experiment Station. 1987. 64 p.

HARRINGTON, T. B. Silvicultural approaches for thinning southern pines: method, intensity and timing. School of Forest Resources, Georgia, EUA. 17 p. 1997.

HUSCH, B.; MILLER, C. I.; BEERS, T. W. Forest mensuration. New York: John Wiley \& Sons, 3 ed., 402 p., 1982.

LIMA, R.; INOUE, M. T.; FIGUEIREDO FILHO, A.; ARAUJO, A. J.; MACHADO, A. S. Efeito do espaçamento no desenvolvimento volumétrico de Pinus taeda L. Floresta e Ambiente, 2013; 20 (2): 223 - 230.

MADRIGAL, A.; Ordenación de Montes arbolados. ICONA Ministerio de Agricultura, Pesca y Alimentación, Colección Técnica. 1994. 375 p.

McCARTER, J. B.; LONG, J. N. A lodgepole pine density management diagram. Western Journal of Applied Forestry. 1986. 1 (1): 6-11. 
NEWTON, P. F. Stand density management diagrams: Review of their development and utility in stand-level management planning. Forest Ecology and Management 98. p. 251 - 265. 1997.

OLIVEIRA, E. B.; OLIVEIRA, Y. M. M.; HAFLEY, W. L. Um Software para Predição do Crescimento e da Produção de Pinus elliottii e Pinus taeda sob manejo no Sul do Brasil. Pesquisa Agropecuária Brasileira, Brasília - DF, v. 26, n. 1, p. 149 - 151, 1991.

REINEKE, L. H. Perfecting a stand-density index for even aged forests. Journal of Agricultural Research, v. 46, n. 7, p. $627-638,1933$.

RETSLAFF, F. A. S. Simulador para prognose da produção de Pinus taeda com Diagrama de Manejo da Densidade associado à modelagem em classes de diâmetro. 166 f. Tese (Doutorado em Engenharia Florestal) - Universidade Federal do Paraná, Curitiba, 2014.

SAUNDERS, M. R.; PUETTMANN, K, J. A preliminary white spruce density management diagram for the lake states. Minnesota: Department of Forest Resources. College of Natural Resources and Minnesota Agricultural Experiment Station. University of Minnesota. 2000. 14 p. (Sraff Paper Series, n. 145).

SCHNEIDER, P. S. P. Autodesbaste e diagrama de manejo da densidade em povoamentos de Pinus taeda L. 94 f. Dissertação (Mestrado em Engenharia Florestal) - Universidade Federal de Santa Maria, Santa Maria, 2008.

SCHNEIDER, P. S. P.; FLEIG, F. D.; SCHNEIDER, P. R.; FINGER; C. A. G.; CHAVES, D. C.; ANTÔNIO MAURÍCIO MOREIRA, A. M. M. Diagrama de manejo da densidade para povoamento de Pinus taeda L. conduzido sem desbaste. Ciência Florestal, Santa Maria, v. 19, n. 4, p. 433 - 447, 2009.

SELlE, G. L.; FlEIG, F. D.; SCHNEIDER, P. R.; ALBERNARD, L. A. J.; VUADEN, E.; BRAZ, E .M. Dendrogramas de densidade para Hovenia dulcis Thunberg na região central do estado do Rio Grande do Sul, Brasil. Ciência Florestal, Santa Maria, v. 20, n. 3, p. 477 - 492, 2010.

TADAKI, Y. The pre-estimating of stem yield based on the competition density effect. Bulletin of the Government Forest Experiment Station (Tokyo, Japan). (In Japanese; English Summary) n. 154. 1963.

VALBUENA, P.; DEL PESO, C.; BRAVO, F. Stand density management diagrams for two mediterranean pine species in eastern Spain. Investigación Agraria: Sistemas y Recursos Florestales, v. 17, n. 2, p. 97 - 104 , 2008. 